Communications in Physics, Vol. 27, No. 4 (2017), pp. 345-355

DOI:10.15625/0868-3166/27/4/10798

\title{
DEVELOPMENT OF UV LASER SOURCE BASED ON DISTRIBUTED FEEDBACK DYE LASERS FOR USE IN MEASUREMENT OF OZONE IN THE LOWER ATMOSPHERE
}

\author{
TIEN PHAM MINH ${ }^{a, b, \dagger}$, HAI BUI VAN ${ }^{c}$, THO DUONG TIEN $^{b}$, \\ $\mathrm{HOA} \mathrm{DO} \mathrm{QUANG}^{d}$ AND TRUNG DINH VAN $^{d}$ \\ ${ }^{a}$ Ho Chi Minh City Institute of Physics, Vietnam Academy of Science and Technology, \\ 1 Mac Dinh Chi Str., Dist. 1, Ho Chi Minh City, Vietnam \\ ${ }^{b}$ Graduate University of Science and Technology, \\ Vietnam Academy of Science and Technology, 18 Hoang Quoc Viet, Cau Giay, Hanoi, Vietnam \\ ${ }^{c}$ Technical University of Le Qui Don, 236 Hoang Quoc Viet, Hanoi, Vietnam \\ ${ }^{d}$ Institute of Physics, Vietnam Academy of Science and Technology, 10 Dao Tan, Ba Dinh, Hanoi, \\ Vietnam \\ ${ }^{\dagger} E$-mail: pmtien@hcmip.vast.vn \\ Received 10 October 2017 \\ Accepted for publication 27 October 2017
}

Published 22 December 2017

\begin{abstract}
We studied to develop a transmitter consisting of two Distributed Feedback Dye Lasers (DFDL) using Rhodamine 6G as an active medium, pumped by a frequency-doubled Nd:YAG laser $(5 \mathrm{~ns}, 10 \mathrm{~Hz}, 532 \mathrm{~nm})$. The BBO crystals are used for frequency-doubling of DFDLs to generate UV emissions. This system has been set up and it generates selected wavelenghts at $282.9 \mathrm{~nm}$ and $286.4 \mathrm{~nm}$ with the energy of $30 \mu \mathrm{J} / \mathrm{pulse}$ and $60 \mu \mathrm{J} / \mathrm{pulse}$ respectively. A DIAL system using this UV transmitter is designed to measure the vertical distribution of ozone with high temporal and spatial resolution. Our simulations of received DIAL signal indicate that the altitude of ozone distribution measurement can reach to over $5000 \mathrm{~m}$ along with range resolution of $100 \mathrm{~m}$.
\end{abstract}

Keywords: DIAL, distributed feedback dye laser, ozone vertical measurement.

Classification numbers: 42.79.Qx; 42.55.Mv; 42.70.Cp.

(C) 2017 Vietnam Academy of Science and Technology 


\section{INTRODUCTION}

In the Earth's atmosphere, most of the ozone (about 90\%) is found in the stratosphere. The remaining ozone is located much nearer the ground in the troposphere. The stratospheric ozone occurs naturally and plays a useful role by absorbing most of the biological damaging UV sunlight. Unlike this, the ozone in the troposphere is man-made. It is formed by the interaction of sunlight with $\mathrm{NO}_{x}$ or VOC (Volatile Organic Compounds), which are created by automobile exhaust and emission from gasoline vapors, power plans, refineries and some other industries. In the lower atmosphere, the ozone is a harmful constituent because it damages crop production, forest growth, many common materials and human health. High level of ozone concentration will reduce the air quality and contribute to the greenhouse effect that cause global warming. As a result, continuous monitoring of ozone is of particular interest in the atmospheric research. For measuring the distribution of ozone, the Differential Absorption LIDAR (DIAL) (LIDARLight Detection and Ranging) technique has been successfully used in the ultraviolet spectral range for several decades as a complement to satellite and ozonesonde measurements [1-6]. The main advantages of DIAL remote sensing technique are the direct determination of the ozone number density with high temporal and spatial resolution but without any calibration. Moreover, DIALs can provide long-term observations, characterize short-term ozone variations and acquire ozone profiles continuously under both daytime and nighttime conditions. The DIAL technique, as described by various researchers [1,7-9], requires the simultaneous emission of two (or more) separate laser beams, but closely spaced wavelengths, in the Hartley-Huggins ozone absorption band $(185-320 \mathrm{~nm})$. They are chosen such that one wavelength is strongly absorbed by ozone $\left(\lambda_{o n}\right)$ and the other located at lower absorption portion of the spectrum $\left(\lambda_{\text {off }}\right)$.

The DIAL technique in the UV wavelength range has been developed using different laser sources for ozone measurements in troposphere. They can be a frequency-quadrupled Nd:YAG laser using in a Raman DIAL [10,11], a fixed-frequency UV laser such as an $\mathrm{KrF}$ excimer laser [9, 12], a tunable laser such as a dye or solid state laser $[5,6,13,14]$. In order to easily select the appropriate emitted wavelengths in the range of 270-300 nm, the dye lasers combined with frequency-doublers have been usually used. The wavelength selection and spectral narrowing in these dye lasers are made by using a plane optical grating at Littrow incidence. This allows to attain dye laser radiations with a narrow spectral width, and a tunable wavelength range of 5-7 nm.

This paper presents a description of new UV laser source used for a DIAL's transmitter. This source consists of two Distributed Feedback Dye Laser (DFDL) and the frequency-doubling BBO crystals for UV emmission of $\lambda_{\text {on }}$ and $\lambda_{\text {off }}$ wavelengths. Such a transmitter for DIAL configuration is the first realization in pratice. The DFDLs have been chosen instead of the dye lasers with the Littrow configuration because of their larger tunning $(10-20 \mathrm{~nm})$ helping to easly select the special emitted wavelengths and theirbetter frequency-doubling efficiency due to ultrashort pulse duration ( $\sim$ picosecond). The narroow bandwidth ( $\sim$ picometer) of the DFDLs is also appropriate to the monochromatic wavelength requirement of a LIDAR system. The parameters of these DFDLs will be used to simulate backscattered signals of a DIAL for measuring tropospheric ozone.

\section{DEVELOPMENT OF UV DIAL'S TRANSMITTER BASED ON DFDLS}

For measuring the ozone concentration in the lower atmosphere, an emitting wavelength pair of the DIAL's transmitter has been chosen at $282.9 \mathrm{~nm}\left(\lambda_{o n}\right)$ and $286.4 \mathrm{~nm}\left(\lambda_{\text {off }}\right)$. This pair is 
located in the Harley absorption band of ozone (Fig. 1). It is the optimal choice when we balance the following considerations: the maximum measurable altitude, the impact of aerosol differential backscattering, the absorption arising from non-ozone species, the impact of solar bachground, the DIAL configuration, including output power, the fluorescence efficiency of the dye used for the active medium and the telescope mirror size.



Fig. 1. Absorption bands of ozone with wavelength pair of the DIAL's transmitter.

We have developed a transmitter which comprises of two DFDLs. A Distributed Feedback Dye Laser needs a photo-induced periodic structure in the lasing material. This is achieved by dividing a pump laser beam into two parts and then these parts are recombined at the dye cell to form an interference pattern, where the periodic change of the gain and refraction index is created. This structure can be considered as a set of semitransparent mirror that form a distributed feedback oscillator or a microcavity in the dye solution. The laser generation wavelength of a DFDL satisfies the Bragg scattering condition and is given by the relation: $\lambda_{i}=\left(\lambda_{p} n\right) /(m \sin \theta)$, where $m$ is the order of the Bragg scattering, $n$ is the refractive index of laser medium, $\lambda_{p}$ is the wavelength of pump laser and $\theta$ is the incidence angle of the pump beam. From the above formula, we find that the wavelength of the DFDL can be easily controlled by changing $\theta$ angle.

Two DFDLs of the DIAL transmitter are designed similarly using Rhodamine 6G as an active medium. A schematic diagram of the DFDL system is shown in Fig. 2. A nanosecond Nd:YAG laser (Quantel Brilliant, pulse duration $5 \mathrm{~ns}$, repetition rate $10 \mathrm{~Hz}$, energy $48 \mathrm{~mJ} / \mathrm{pulse}$ ) is employed as a pumping source of two DFDLs with a beam splitter $(R: T=50: 50)$. Each DFDL includes a distributed feedback oscillator $\mathrm{C} 1$, a multipass amplifier $\mathrm{C} 2$ to obtain a much higher gain of DFDL beam, and a power amplifier C3. The cells C1, C2 and C3 are the quartz flowing cuvettes contained circulation mixed dye solution Rh6G in ethanol solvent with $10^{-3} \mathrm{~mol} / \mathrm{L}, 5.10^{-4} \mathrm{~mol} / \mathrm{L}$ and $10^{-4} \mathrm{~mol} / \mathrm{L}$ concentration, respectively. Table 1 lists the component characteristics of a DFDL.

By tilting the angle of the mirrors $\mathrm{m} 1$ and $\mathrm{m} 2$ that direct the pump beams onto the dye solution, the $\theta$ angles are selected appropriately so that DFDLs can emit the radiations at the wavelengths of $565.8 \mathrm{~nm}$ and $572.6 \mathrm{~nm}$. After passing through the BBO crystals of these radiations, a 


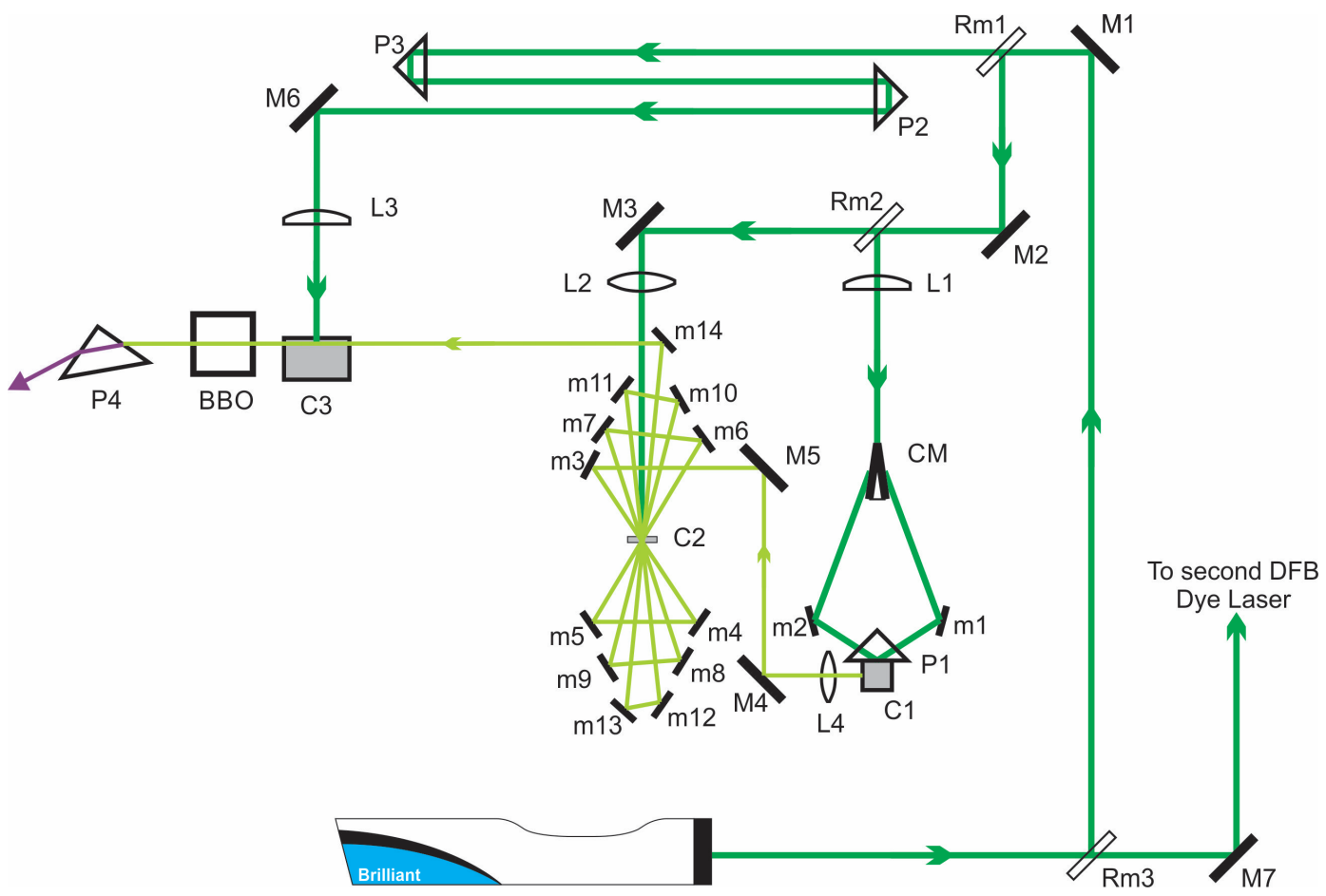

Fig. 2. DFDL diagram. The components in this diagram are listed in Table 1.

Table 1. Characteristics of optical elements of the DFDLs.

\begin{tabular}{lll} 
C1, C2, C3 & Quartz flow cuvette cell & $10 \mathrm{~mm}, 1 \mathrm{~mm}, 20 \mathrm{~mm}$ \\
Rm1, Rm2 & Beamsplitter & $20: 80(\mathrm{R}: \mathrm{T})$ \\
M1 - M6 & Mirrors & $\varphi \quad 1 ”$ \\
m1 - m14 & Mirrors & Square $1 \times 1(\mathrm{~cm})$ \\
CM & Couple mirror & $30^{\circ}$ (angle between the two mirrors) \\
L1, L3 & Plano-convex cylindrical lenses & $f=25 \mathrm{~cm}, 15 \mathrm{~cm} ; 20 \times 22 \mathrm{~mm}$ \\
L2, L4 & Plano-convex spherical lenses & $f=10 \mathrm{~cm}, 2.5 \mathrm{~cm} ; \varphi=1 ”, 0.5^{\prime \prime}$ \\
P1, P4 & Right-angle Prisms & UV- Fused silica, $20 \mathrm{~mm}$ \\
P2, P3 & Right-angle Prisms & N=BK7, $15 \mathrm{~mm}$ \\
\hline
\end{tabular}

CCS200 Spectrometer (Thorlabs), which is calibrated by the spectrum of a Na lamp, indicates that the actual wavelengths of the outgoing UV lasers are $282.9 \mathrm{~nm}$ and $286.4 \mathrm{~nm}$, respectively within an uncertainty of $0.2 \mathrm{~nm}$ (Fig. 3). 


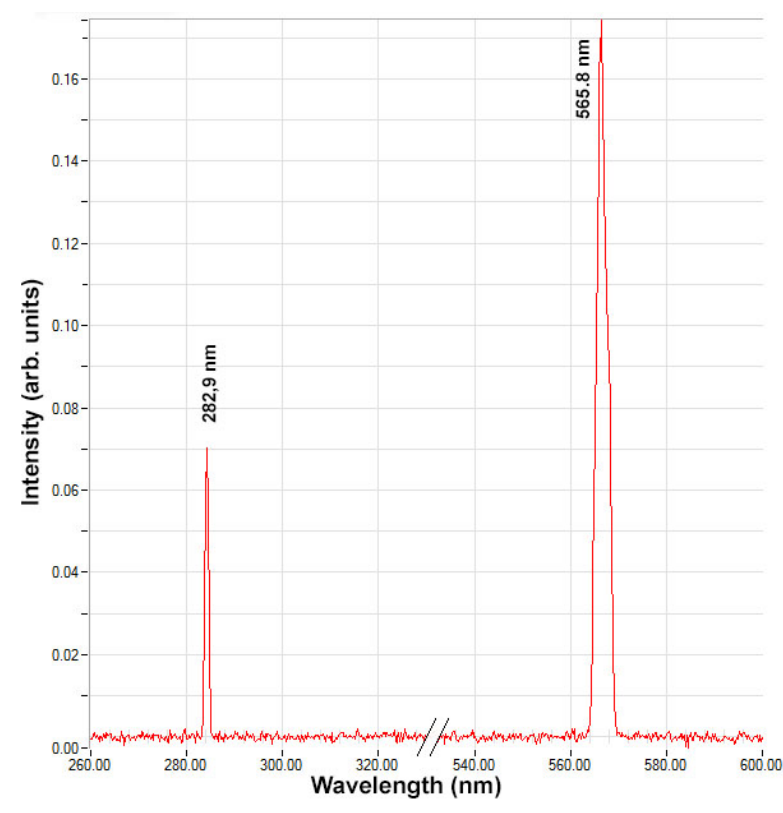

(a)

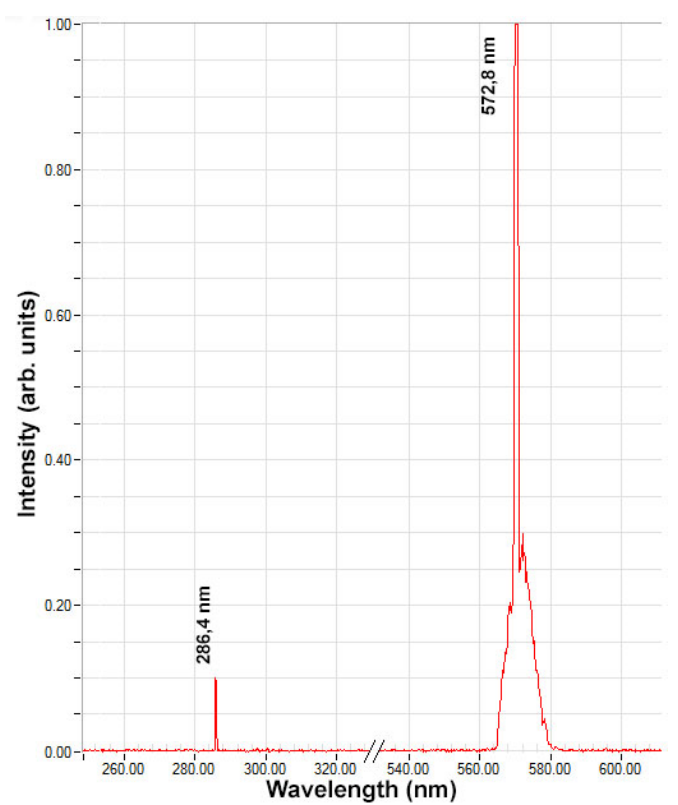

(b)

Fig. 3. (a) UV $\lambda_{\text {on }}$ and DFDL's wavelength at $282.9 \mathrm{~nm}$ and $565.8 \mathrm{~nm}$; (b) UV $\lambda_{\text {off }}$ and DFDL's wavelength at $286.4 \mathrm{~nm}$ and $572.8 \mathrm{~nm}$.

Table 2. Main parameters of our transmitter.

\begin{tabular}{lll}
\hline Transmitter & \multicolumn{1}{c}{ Specification } \\
\hline Pump laser & $\begin{array}{l}\text { Quantel Brilliant Nd:YAG laser, pulse duration } 5 \mathrm{~ns}, \\
\text { repetition rate } 10 \mathrm{~Hz}, \text { energy } 48 \mathrm{~mJ} / \text { pulse }\end{array}$ \\
\hline DFDL & $\begin{array}{l}\text { Rhodamine } 6 \mathrm{G}\left(10^{-3} \mathrm{~mol} / \mathrm{L}\right), \text { spectral width } 14 \pm 2 \mathrm{pm}, \\
\text { duration of pulse } 20 \pm 2 \mathrm{ps}\end{array}$ \\
\hline & $565.8 \mathrm{~nm}$, energy $0.62 \mathrm{~mJ} / \mathrm{pulse}$ & $572.8 \mathrm{~nm}$, energy $1.8 \mathrm{~mJ} / \mathrm{pulse}$ \\
\hline Emitted UV & $\begin{array}{l}30 \mu \mathrm{J} / \text { pulse at } 2829 \mathrm{~nm}, \\
\text { divergence }<2 \mathrm{mrad}\end{array}$ & $\begin{array}{l}60 \mu \mathrm{J} / \text { pulse at } 2864 \mathrm{~nm}, \\
\text { divergence }<2 \mathrm{mrad}\end{array}$ \\
\hline
\end{tabular}

A Power Meter (OPHIR Nova II - Newport) was employed to measure the energy of DFDL pulses. The spectral width of the emission of the DFDLs was measured by using a Fabry-Perot $(\mathrm{F}=35)$. In addition, the stability of the UV transmit power was investigated. A photodiode (S1226-18BU-Himamatsu) was used to measure the average intensity of UV laser pulses. The results of UV intensity measurement indicate that the power fluctuation of UV pulses is less than $1.2 \%$ over a 20 minute period. The power fluctuation and the deterioration of UV power pulses 
are acceptable and do not affect the result of differential calculations for retrieval of ozone density. The main transmitting system parameters are summarized in Table 2.

\section{UV DIAL SYSTEM DESIGN FOR MEASURING TROPOSPHERIC OZONE}

Based on a transmitter emitting two radiations at $282.9 \mathrm{~nm}$ and $286.4 \mathrm{~nm}$ wavelengths, a design of the UV DIAL system for measuring tropospheric ozone is proposed. This DIAL configuration is shown in Fig. 4.

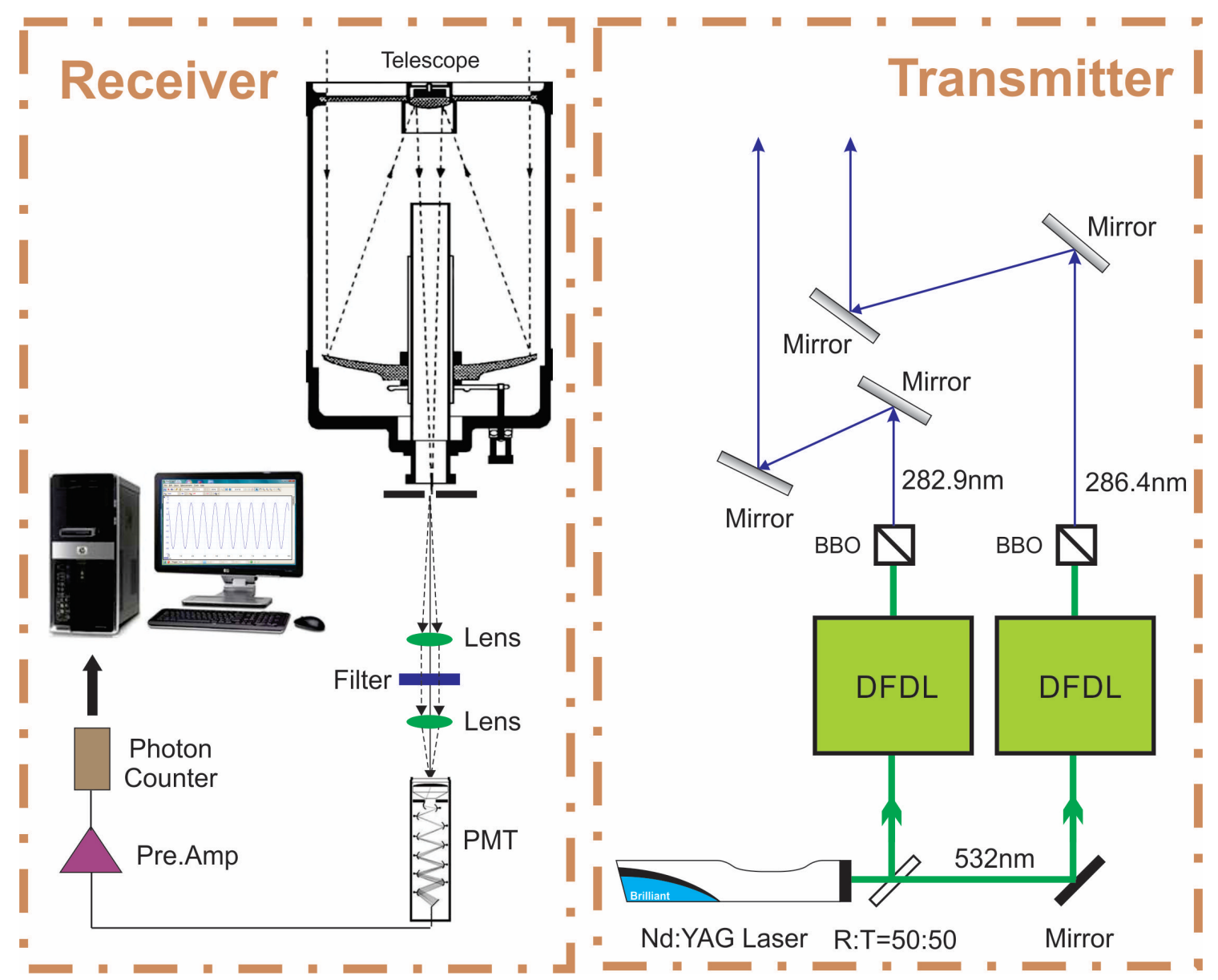

Fig. 4. Diagram of UV DIAL for measuring tropospheric ozone.

The DIAL system includes a transmitter, a receiver and a detection system of the DIAL signals. The transmitter consists of two DFDLs, pumped by a frequency-doubled Nd:YAG laser. The BBO crystals are used for doubling of pulse of DFDLs to emit two UV radiations at $\lambda_{\text {on }}$ and $\lambda_{\text {off }}$ wavelengths. The receiver operates with a telescope and an optical system for filtering elastic backscattering signals. The detection system uses a photomultiplier tube (PMT), a preamplifier (Pre.Amp.), a photon counter and a computer to acquire these signals. 


\section{SIMULATION OF THE RECEIVED BACKSCATTERED DIAL SIGNALS}

We have simulated return signals as a function of range by using Matlab software to estimate the expected LIDAR signals of the designed DIAL system. The simulation results are important as they can be used for assessment of the potential of this DIAL system, for comparisons with experimental measurements, and for optimizing our designed system. Following the LIDAR equation [1], $P(R, \lambda)$-LIDAR return signal power at wavelength $\lambda_{\text {on }}$ or $\lambda_{\text {off }}$ is given by:

$$
\begin{gathered}
P\left(R, \lambda_{o n}\right)=P_{0}\left(\lambda_{o n}\right) \frac{c \tau}{2} A \eta \frac{O(R)}{R^{2}} \beta\left(R, \lambda_{o n}\right) \exp \left[-2 \int_{0}^{R} \alpha\left(r, \lambda_{o n}\right) d r\right] \\
P\left(R, \lambda_{o f f}\right)=P_{0}\left(\lambda_{o f f}\right) \frac{c \tau}{2} A \eta \frac{O(R)}{R^{2}} \beta\left(R, \lambda_{o f f}\right) \exp \left[-2 \int_{0}^{R} \alpha\left(r, \lambda_{o f f}\right) d r\right],
\end{gathered}
$$

where $R$ is the distance between the backscattering atmospheric layer and the telescope, $P_{0}(\lambda)$ is the UV laser power emitted by DIAL system, $\eta$ is the overall system efficiency, $\tau$ is the temporal pulse length, $A$ is the area of the light collection, c is the speed of the light, $O(R)$ is laser-beam receiver-field-of-view overlap function, $\beta(R, \lambda)$ is the backscatter coefficient, $\alpha(r, \lambda)$ is the extinction coefficient at wavelength $\lambda$ and range $r$.

Since the distance between $\lambda_{\text {on }}$ and $\lambda_{\text {off }}$ wavelengths is small, the system efficiency $\eta$ is given the same value in the equation (1) and (2). $\beta(R, \lambda)$ is the sum of the molecular (mol) and aerosols (aer) backscattering coefficients of the atmosphere:

$$
\beta(R, \lambda)=\beta_{\text {mol }}(R, \lambda)+\beta_{\text {aer }}(R, \lambda)
$$

$\alpha(\mathrm{r}, \lambda)$ is the extinction coefficient of the air due to the molecular (Rayleigh) scattering, to the aerosols (Mie) scattering and absorption, and to the absorption (abs) of ozone:

$$
\alpha(r, \lambda)=\alpha_{m o l}(r, \lambda)+\alpha_{a e r}(r, \lambda)+\alpha_{O_{3}, a b s}(r, \lambda)
$$

For this simulation, the absorption of other molecules than ozone $\left(\mathrm{O}_{2}, \mathrm{NO}_{2}\right.$ and $\left.\mathrm{SO}_{2}\right)$ is assumed to be very small and can be ignored. Based on the results of the atmospheric aerosol measurement over Hanoi [15], some ratios have been supposed for solving the LIDAR equation. The LIDAR ratio (aerosol extinction-to-backscatter ratio) $L_{a e r}=\alpha_{a e r}(R) / \beta_{a e r}(R)$ is assumed to be known and is equal to 30 for the $286.4 \mathrm{~nm}\left(\lambda_{\text {off }}\right)$ signal. $\mathrm{L}_{a e r}$ depends on the size distribution, shape, humidity and chemical composition of the aerosols $[1,2]$. The aerosol-to-molecular backscatter ratio at the offline $\lambda_{\text {off }}$ wavelength is defined as $B=\beta_{a e r} / \beta_{\text {mol }}$. Under clear sky condition in Hanoi, the value of $B$ is usually equal to several units for $5 \mathrm{~km}$ above the ground. For simplicity, we assume that B is estimated to be 3 for altitudes below $5 \mathrm{~km}$. The parameters using in simulation calculation are the experimental parameters of the designed DIAL system and they are shown in Table 3. The simulation of backscattering and extinction coefficients have used the atmospheric parameters retrieved from Aero-Meteorological Observatory in Lang, Hanoi: vertical distributions of nitrogen, oxygen and ozone. 
DEVELOPMENT OF UV LASER SOURCE BASED ON DISTRIBUTED FEEDBACK DYE LASERS FOR USE ...

Table 3. Parameters of UV DIAL system used for simulations.

\begin{tabular}{|c|c|c|}
\hline \multirow{4}{*}{ Transmitter } & Laser radiation at $\lambda_{\text {on }}$ wavelength & $282.9 \mathrm{~nm} ; 30 \mu \mathrm{J} /$ pulse \\
\hline & Laser radiation at $\lambda_{\text {off }}$ wavelength & $286.4 \mathrm{~nm} ; 60 \mu \mathrm{J} /$ pulse \\
\hline & Laser repetition rate & $10 \mathrm{~Hz}$ \\
\hline & Optical efficiency & $77 \%$ at $\lambda_{o n}$ and $\lambda_{o f f}$ \\
\hline \multirow{4}{*}{ Optical Receiver } & Telescope & $40 \mathrm{~cm}$ and $60 \mathrm{~cm}$ diameter \\
\hline & Reflectance of telescope & $60 \%$ at $\lambda_{o n}$ and $\lambda_{o f f}$ \\
\hline & Transmittance of Lens & $93 \%$ at $\lambda_{o n}$ and $\lambda_{o f f}$ \\
\hline & Filter efficiency & $82 \%$ at $\lambda_{\text {on }}$ and $\lambda_{o f f}$ \\
\hline \multirow{3}{*}{ Data acquisition system } & Optical eff. to PMT & $70 \%$ at $\lambda_{o n}$ and $\lambda_{o f f}$ \\
\hline & Sampling frequency & $42 \mathrm{M}$ samples/s \\
\hline & Dart counts rate of PMT & 100 counts $/ \mathrm{s}$ \\
\hline
\end{tabular}

Since the DIAL operating in the photon counting mode, the number of signal pulses counted can be expressed as a Poisson distribution. Thus the signal-to-noise $(\mathrm{S} / \mathrm{N})$ ratio is as follows [16]:

$$
S / N=\frac{N_{p h}}{n_{t o t}}=\frac{N_{p h}}{\sqrt{N_{p h}+N_{b}+N_{d}}}
$$

where $N_{p h}$ is the number of counts by signal light, $N_{b}$ is the number of counts by background light, $N_{d}$ is the number of dark counts, and $n_{t o t}$ is total noise. In ultraviolet DIAL case, we can skip the $N_{b}$ due to its negligible quantity.

The photon number backscattered by atmospheric molecules when two pulsed laser beams at $282.9 \mathrm{~nm}$ and $286.4 \mathrm{~nm}$ are sent into the atmosphere has been calculated and shown in Fig. 5. In this simulation, the LIDAR equation has been computed with the presence of the extinction due to ozone absorption and with telescope diameter of $40 \mathrm{~cm}$ of the receiver. Currently, we are developing a telescope with a diameter of $60 \mathrm{~cm}$. The simulation results indicate that if this $60 \mathrm{~cm}$ diameter telescope is used, the number of photons received by designed DIAL system at $\lambda_{\text {on }}$ and $\lambda_{\text {off }}$ wavelengths will be increased by 2.5 times (see Fig. 6).

Fig. 7 shows the $\mathrm{S} / \mathrm{N}$ ratios vs. altitude at $\lambda_{o n}$ and $\lambda_{\text {off }}$ wavelengths. If 3 is the best $\mathrm{S} / \mathrm{N}$ ratio that we can distinguish the signals from the noises, we can retrieve LIDAR signals from the altitude over $5000 \mathrm{~m}$ at range resolution of $100 \mathrm{~m}$. 


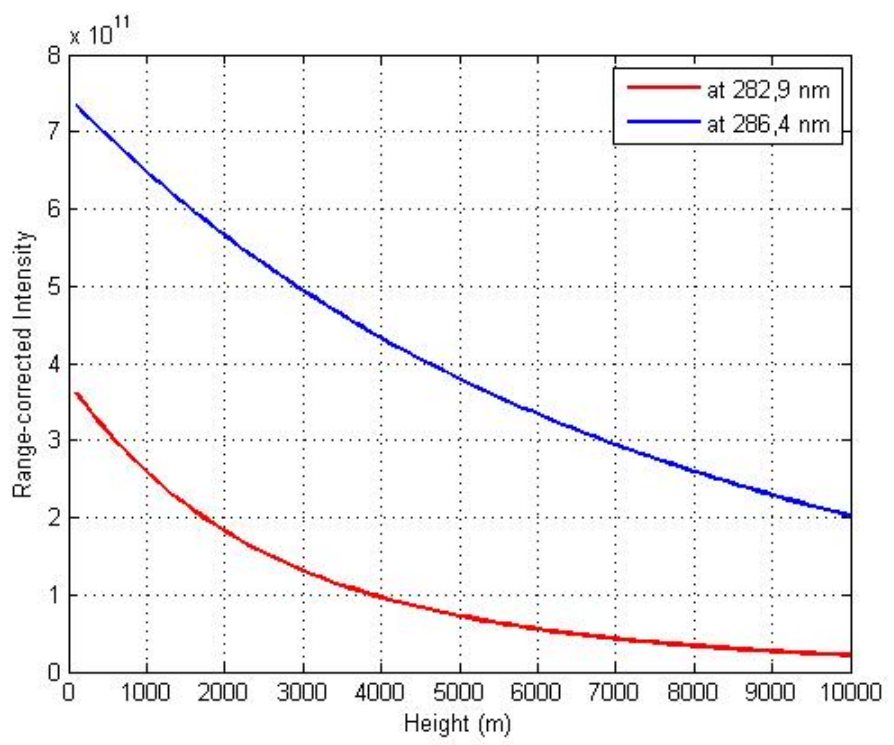

Fig. 5. Simulation of the number of photons backscattered by atmospheric molecules at $\lambda_{\text {on }}$ and $\lambda_{\text {off }}$ wavelengths with range resolution of $100 \mathrm{~m}$ and counting duration of $10 \mathrm{~min}$.

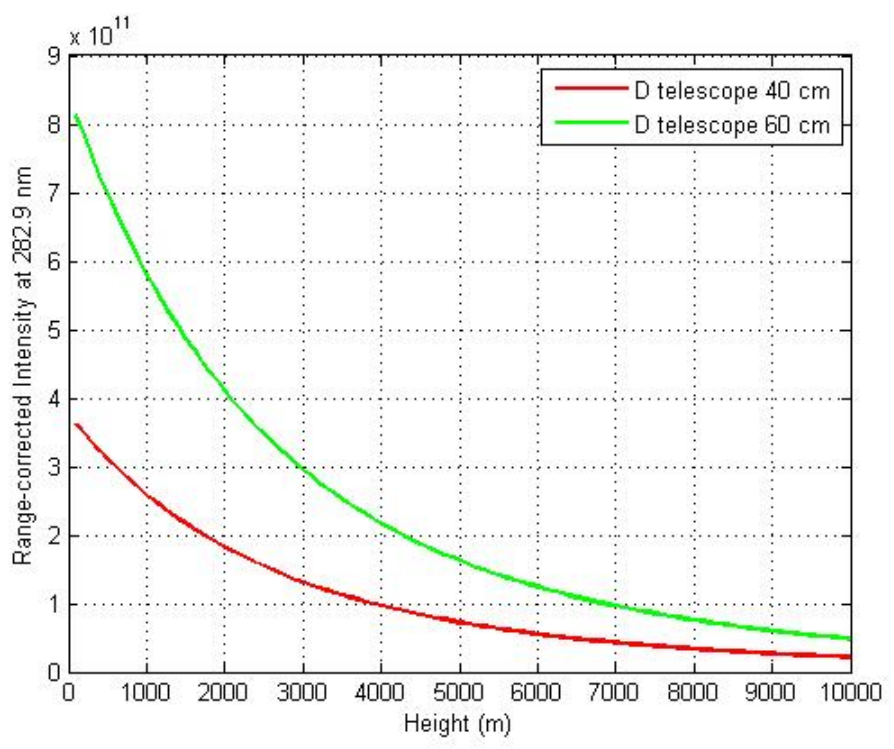

Fig. 6. Simulation of the number of photons backscattered by atmospheric molecules at $\lambda_{\text {on }}$ wavelength in two cases of the telescope diameter: $40 \mathrm{~cm}$ and $60 \mathrm{~cm}$ with range resolution of $100 \mathrm{~m}$ and counting duration of $10 \mathrm{~min}$. 


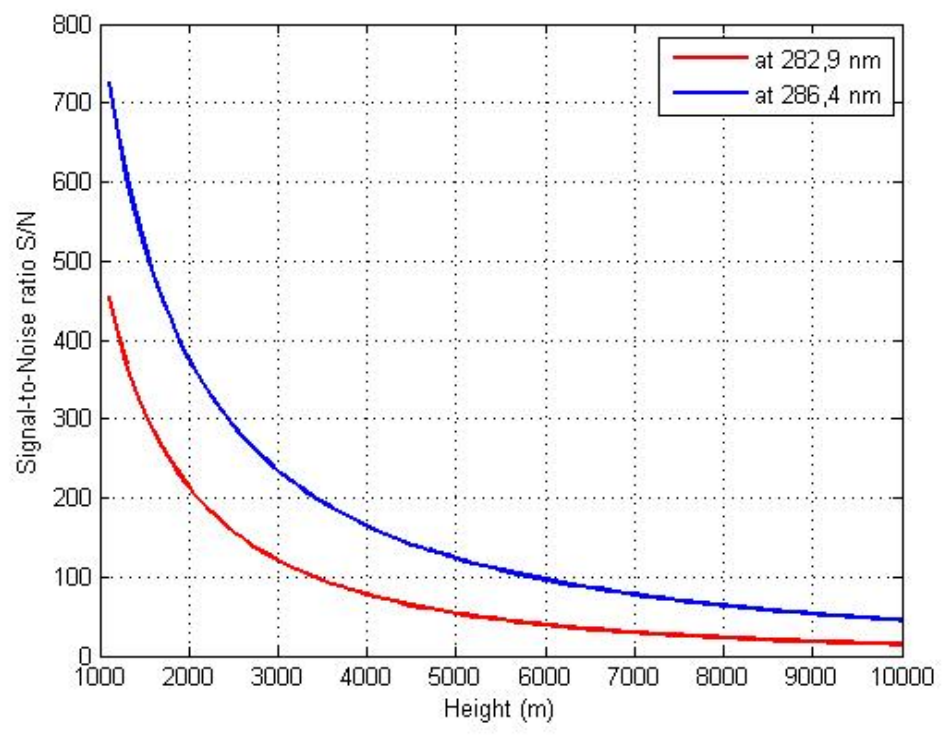

Fig. 7. S/N ratio vs. altitude at $\lambda_{o n}$ and $\lambda_{o f f}$ wavelengths.

\section{CONCLUSION}

We have developed a laser transmitter to be used in a DIAL system. This transmitter comprises two DFDLs pumped by a frequency-doubled Nd:YAG laser $(5 \mathrm{~ns}, 10 \mathrm{~Hz}, 532 \mathrm{~nm})$ and the BBO crystals to generate the UV wavelengths at $282.9 \mathrm{~nm}$ and $286.4 \mathrm{~nm}$, energy $30 \mu \mathrm{J} / \mathrm{pulse}$ and $60 \mu \mathrm{J} /$ pulse, respectively. A DIAL system using this UV transmitter is designed and simulated to measure the vertical distribution of ozone with high temporal and spatial resolution. Our simulations of backscattered signals indicate that this transmitter is appropriate to a DIAL system which can be used to measure the vertical ozone distribution to an altitude of over $5000 \mathrm{~m}$, with range resolution of $100 \mathrm{~m}$, counting duration of 10 minutes and a receiver using a telescope of $40 \mathrm{~cm}$ diameter. A comprehesive description of this DIAL system and ozone measurement results will be published in the near future.

\section{ACKNOWLEDGMENTS}

The development of this UV DIAL transmitter was supported by the funds from the project VAST01.08/13-14 and from the Vietnam National Foundation for Science and Technology Development (NAFOSTED) under Grant Number 103.99 -2014.82. We would like to thank all our colleagues in the Laboratory for Atmospheric Research (Institute of Physics, VAST) for their assistance in the development of this DIAL system.

\section{REFERENCES}

[1] Claus Weitkamp, Lidar Range-Resolved Optical Remote Sensing of the Atmosphere, Springer, ISBN 0-38740075-3, 2005. 
[2] Shi Kuang, John F. Burris, M. J. Newchurch, Steve Johnson, and Stephanie Long, IEEE transactions on geoscience and remote sensing, 49 (2001) 557.

[3] O. Uchino, M. Maeda, H.Yamamura, M. Hirono,J. of Geophysical Research 88 (1983) 5273.

[4] P. C. S. Devara, P. Ernest Raj, G. Pandithurai, K. K. Dani, S. M. Sonbawne and Y. Jaya Rao, Meas. Sci. Techno. 18 (2007) 639.

[5] Shi Kuang, John F. Burris, M. J. Newchurch, Wesley Cantrell, Guanyu Huang, Ozone Lidar Measurements in the Boundary layer, AGU (American Geophysical Union) Fall Meeting, Sanfrancisco, CA, 2012.

[6] Sung-Chul Choi and Young-Joon Kim, J. of the Korean Physical Society 44 (2004) 1432.

[7] R. M. Schotland, The determination of the vertical profile of atmospheric gases by means of a ground-based optical radar, in Proceedings of the Third Symposium on Remote Sensing of Environment, University of Michigan, Ann Arbor, 1964.

[8] J. Pelon, S. Godin, and G. Mégie, J. Geophys. Res. 91 (1986) 8667

[9] O. Uchino, M. Maeda, H. Yamamura and M. Hirono, J. of Geophysical Research 88 (1983) 5273.

[10] P. Jason Collier, Sameer Unni, Sachin J. Verghese, Adam Willitsford, C. Russell Philbrick, Richard Clark and Bruce Doddridge, Raman Lidar Measurements of Tropospheric Ozone, in Proceedings of the $5^{\text {th }}$ Conference on Atmospheric Chemistry: Gases, Aerosols and Clouds, 2003.

[11] BenoîtLazzarotto, Gilles Larchevêque, Philippe Quaglia, ValentinSimeonov, Hubert Van den Bergh and Bertrand Calpini, "Raman DIAL measurement of ozone and water vapor in the lower troposphere", Proceedings Vol. 3821, Environmental Sensing and Applications, Germany, 1999.

[12] I. Veselovskii and B. Barchunov, Appl. Phy. B 68 (1999) 1131.

[13] E. V. Browell, M. A. Fenn, C. F. Butler, W. B. Grant, R. C. Harriss and M. C. Shipham, J. Geophys. Res. 99 (1994) 1739.

[14] E. Durieux, L. Fiorani, B. Calpini, M. Flamm, L. Jaquet and H. Van Den Bergh, Atmospheric Environment, 32 (1998) 2141.

[15] Bui Van Hai, Dinh Van Trung, Nguyen Xuan Tuan, Nguyen Dinh Hoang, Dam Trung Thong, Nguyen ThanhBinh, Determination of Atmospheric Aerosol Extinction with a Raman LIDAR system over Hanoi, Advances in Optics, Photonics, Spectroscopy \& Applications VII, ISSN 1859-4271, 2013.

[16] Philip B. Russell, Thomas J. Swissler and M. Patrick McCormick, Applied Optics 18 (1979) 3783. 